\title{
Anabases
}

ANABASES Traditions et réceptions de l'Antiquité

23 | 2016

Varia

\section{Une traversée des conjonctures intellectuelles,} 1970-2015

Entretien avec François Hartog

\section{CpenEdition}

Journals

Édition électronique

URL : http://journals.openedition.org/anabases/5616

DOI : 10.4000/anabases.5616

ISSN : 2256-9421

Éditeur

E.R.A.S.M.E.

Édition imprimée

Date de publication : 2 mai 2016

Pagination : 115-136

ISSN : 1774-4296

Référence électronique

« Une traversée des conjonctures intellectuelles, 1970-2015 », Anabases [En ligne], 23 | 2016, mis en ligne le 02 juin 2019, consulté le 21 octobre 2019. URL : http://journals.openedition.org/anabases/ 5616 ; DOI : 10.4000/anabases.5616 
Anabases 23 (20і6), p. пІ5-І36.

\section{Une traversée des conjonctures intellectuelles, 1970-2015'}

Entretien avec François Hartog

I'invitation de l'équipe ERASME (Université Toulouse - Jean Jaurès) s'est tenue,
le 7 octobre 2oI5, une table ronde destinée aux travaux et aux recherches de
François Hartog. À la fois historien et voyageur, François Hartog consacre ses premières enquêtes à l'Odyssée, puis une thèse d'État à Hérodote, d'où est issu un livre : Le miroir d'Hérodote. Essai sur la représentation de l'autre (Gallimard, Ig8o) qui devient vite un repère majeur dans le champ de l'historiographie et dans celui des rapprochements entre histoire et anthropologie. François Hartog enseigne l'histoire grecque aux universités de Strasbourg et de Metz, puis est élu directeur d'Études à l'École des Hautes Études en Sciences Sociales, à Paris (I987). Il y développe des recherches fondées sur les rapports entre histoire et historiographie, entre Anciens et Modernes, toujours attentif aux conjonctures qui traversent les sciences humaines et sociales. De là est issue une quinzaine d'ouvrages qui sont pour la plupart mentionnés dans l'entretien ci-dessous et qui constituent une des réflexions majeures de notre temps sur le rôle de l'histoire, les fonctions et l'identité de l'historien, les interférences de la mémoire et du temps de l'histoire, en particulier dans Mémoire d’Ulysse. Récits sur la frontière en Grèce ancienne (Gallimard, Ig96), Anciens, Modernes, Sauvages (Galaade Éditions, 2005, “Points » Seuil, 2008), Croire en l'histoire (Flammarion, 20I3), Partir pour la Grèce (Flammarion, 20I5).

1 L'enregistrement de cette table ronde a été retranscrit par Anthony Andurand et Clément Bertau, révisé par Corinne Bonnet et Pascal Payen, amendé par François Hartog, que nous tenons à remercier très chaleureusement pour la générosité dont il a fait preuve au cours des deux journées passées à Toulouse. 
Pascal Payen: En préambule à cette table ronde, dont vous avez choisi le titre, “Une traversée des conjonctures intellectuelles, I970-20I5 », j’aimerais vous demander quelle signification vous donnez à “ conjonctures intellectuelles », avec l'image de la «traversée, qui fait écho au titre du dernier livre de Jean-Pierre Vernant».

François Hartog: Je pense que l'expression de “conjonctures intellectuelles» est elle-même assez claire. Le problème des conjonctures intellectuelles, comme de toute chose, c'est qu'on ne peut les dessiner ou les circonscrire qu'après. Quand on est dedans, on a du mal à s'y retrouver. C'est l'expérience, au fond, que chacun fait. Si vous vous demandez, vous qui êtes étudiants - plus ou moins avancés dans votre cursus -, quelle est la conjoncture intellectuelle dans laquelle vous vous trouvez au moment où vous engagez vos études, vous auriez peut-être quelques difficultés à répondre à cette question. Cette difficulté concerne tout le monde et toutes les époques, mais il me semble qu'entre la fin des années ig6o et le début du $\mathrm{xxI}^{\mathrm{e}}$ siècle, nous avons traversé des conjonctures intellectuelles différentes. Pour le dire de façon schématique, quand j'ai commencé à travailler et à réfléchir sur la conjoncture intellectuelle, nous étions dans ces années que l'on peut caractériser par quelques noms: ceux de Claude Lévi-Strauss, de Michel Foucault ou de Roland Barthes, mais aussi celui de Jean-Pierre Vernant et de ceux qui avaient commencé à travailler avec lui. Je ne peux les énumérer tous, mais il y avait quelques noms repères. Ces années, pour nous qui découvrions alors la vie intellectuelle et politique, se résument en un mot: le structuralisme, avec toutes les ambiguïtés, les confusions qu'il pouvait comporter. C'était une sorte de mot d'ordre, d'étiquette, de mot de combat, aussi, puisqu'il s'agissait de revendiquer le structuralisme sans même savoir ce que c'était. C'était s'opposer à l'enseignement que nous recevions alors: d'un côté, la Sorbonne et, de l'autre, des espaces qui développaient une perspective critique sur ce qui constituait et qui était présenté comme l'enseignement le plus traditionnel. Et cela était encore plus vrai, peutêtre, dans le domaine des études classiques.

Alors «traverser », c'est justement le fait que volens nolens on passe au travers de ces conjonctures, mais que l'on essaye aussi de saisir le passage d'une conjoncture à une autre. C'est quelque chose qui m'a toujours non seulement intéressé, mais profondément mobilisé et inquiété: savoir ce qu'il est en train de se passer. Au fond, si je rapporte cela au travail que j'ai pu faire, Le miroir d'Hérodote ${ }^{2}$ est un peu à la jonction, non de deux conjonctures, mais de deux moments d'une conjoncture. Il y a, d'une part, le structuralisme et l'anthropologie, mais aussi, d'autre part, les interrogations sur le récit et sur l'écriture de l'histoire. Si je prends deux noms qui

2 F. Hartog, Le Miroir d'Hérodote. Essai sur la représentation de l'autre, Paris, Gallimard, $200 \mathrm{I}^{3}[\mathrm{I} 98 \mathrm{o}]$. 
marquaient alors le paysage, j'évoquerais Ferdinand de Saussure et son approche par la langue, ainsi qu'Émile Benveniste, le grand linguiste, qui commençait à revenir sur le problème de la langue à travers une interrogation sur l'énonciation, c'està-dire sur les modalités d'intervention du narrateur dans son récit. Pour ce qui est de l'interrogation sur l'anthropologie, il y a bien sûr l'intervention de Lévi-Strauss et l'anthropologie historique proposée par Vernant et son entourage. J'ajouterais le nom de Michel de Certeau, qui faisait intervenir une dimension réflexive sur, précisément, la manière dont on écrit l'histoire.

Pascal Payen: Pour rester dans ce contexte, je voudrais aussi vous interroger, dans un premier temps, sur l'ensemble de votre parcours personnel d'historien et d'intellectuel, tel qu'il apparaît au fil des quatorze livres que vous avez écrits. Je le ferai sans entrer dans le registre convenu de l'ego-histoire et de la vulgate qui l'accompagne souvent maintenant. Pour cela, je m'abriterai derrière un mot que vous formulez vous-même au sujet de Fustel de Coulanges, l'auteur en i864 de La cité antique: ‘Fustel de Coulanges a travaillé sur l'Antiquité: ses premiers travaux lui sont consacrés. Mais jamais, avant même de choisir l'histoire de la France, il ne s'est limité au monde antique ${ }^{3}$. Vous avez vous aussi commencé par vous intéresser à l'Antiquité, aux Phéaciens dans un mémoire de maîtrise, et puis à Hérodote, objet d'une thèse d'État. Qu'est-ce qui a déclenché en vous cette envie de ne pas en rester là, de ne pas en rester à l'Odyssée et à Hérodote, qui auraient pu suffire à toute une vie de chercheur? En quoi la conjoncture a-t-elle fait bifurquer votre chemin? À quoi, finalement, aspiriez-vous intellectuellement à ce moment-là?

François Hartog: On peut s'occuper pendant quelques années avec Homère et Hérodote, c'est certain... Mais je ne l'ai pas fait. Même si Ulysse m'a accompagné un bon bout de temps. Pour répondre à votre question, il y a d'abord un élément factuel: les gens avec qui j'ai eu la chance de travailler, à savoir Vernant et Vidal-Naquet. L'un et l'autre, de manière différente d'ailleurs, n'ont jamais été seulement des antiquisants. Vernant, par sa biographie, a été un militant, un résistant, quelqu'un qui a participé pendant des années à son contemporain. Et même lorsqu'il a fait le choix de la Grèce, de “partir » véritablement pour la Grèce, il a continué à avoir d'autres engagements, d'autres activités, par exemple avec la Russie, avec les dissidents. Vernant, donc, a su à la fois se donner les moyens d'être un helléniste - même si on lui a toujours reproché de ne pas connaître le grec - et ne jamais renoncer à être lui-même. Quant à Vidal-Naquet, il a toujours voulu faire

3 F. Hartog, Le xix siècle et l'histoire. Le cas Fustel de Coulanges, Paris, Éditions du Seuil, $200 \mathrm{I}^{2}[\mathrm{I} 988]$, p. 33 . 
autre chose que de l'histoire ancienne. Comme il l'explique dans ses Mémoires ${ }^{4}$, il est toujours revenu à l'histoire ancienne, mais il n'a cessé d'en sortir. Il était complètement pris, quelques fois obsédé, par ce qui se passait. Vous n'avez pas pu connaître sa manière d'être un intellectuel, ses interventions multiples dans les journaux, en particulier dans Le Monde. Pour lui, faire un article à la hâte sur tel ou tel problème concernant les Palestiniens, concernant telle autre injustice, c'était absolument requis. C'était sa manière d'être. Dans le livre que je lui ai consacrés,je me suis interrogé, précisément, sur cette façon d'être au monde, qui pour lui était toujours une façon d'être historien. Tout le livre porte au fond sur cette question: il affirmait que tout ce qu'il avait fait, il l'avait fait «en tant qu'historien». Que veut dire en tant qu'historien? Quelqu'un qui peut aussi bien vous parler d'un bordereau d'ensemencement, de Flavius Josèphe, de Clisthène, de la Révolution française, que de telle rencontre avec Arafat.

Il y a donc, d'abord, des éléments factuels: les gens avec qui j'ai travaillé, le milieu dans lequel je me trouvais, le groupe qui m’a accueilli. Si je me suis trouvé là, si j’ai choisi ce groupe-là, me direz-vous, ce n'est pas non plus complètement par hasard. C'est bien que cette manière d'être au monde me préoccupait, et donc je me suis efforcé, différemment d'eux-mêmes il est vrai, de ne jamais m'en tenir à la seule Antiquité. Non que je considère que c'est insuffisant pour remplir une vie, mais le rapport à l'Antiquité passe par cet effort de saisir ce qui est en train de se passer.

Pascal Payen: Ma seconde question d'ensemble touche à un domaine que je qualifierai de «longue durée»: c'est la question de l'historiographie comme discipline. Dans la préface d'un tout récent Dictionnaire de l'historien, les directeurs du volume écrivent que «l'histoire, au bout du compte, n'existe que parce qu'ellemême a une histoire, sans cesse remise sur le métier ${ }^{6}$ ». Je voudrais donc vous demander si, au cours des dernières décennies et notamment depuis les années I970, l'historiographie a, selon vous, vraiment intégré le champ des disciplines historiques, et plus largement le champ des disciplines des sciences humaines et sociales? Et, question complémentaire, quelles sont les principales conséquences de cette intégration, ou du refus que l'on a parfois opposé à cette discipline naissante?

4 P. Vidal-Naquet, Mémoires, I. La brisure et l'attente Ig3o-Ig55, 2. Le trouble et la lumière Ig55-ı998, Paris, La Découverte, I995-ı998.

5 F. Hartog, Vidal-Naquet, historien en personne. L'homme-mémoire et le momentmémoire, Paris, La Découverte, 2007.

6 Cl. Gauvard, J.-F. Sirinelli, “Introduction », in Dictionnaire de l'historien, Paris, puf, 20I5, p. XIII. 
François Hartog: La réponse à votre double question ne saurait être tranchée. Ce serait trop simple. Elle se rattache, aussi, à ces courbes qui caractérisent les évolutions de la discipline et que l'on pourrait tracer au fil des décennies. Il me semble que l'approche historiographique, au sens d'une histoire de l'histoire, émerge en France aux alentours des années I980, et guère avant. Un des signes que l'on peut retenir de cette émergence, c'est la traduction chez Gallimard, en I983, d'un recueil de textes d'Arnaldo Momigliano ${ }^{7}$. Ces textes ont été écrits bien avant cette date, mais ils ont alors été traduits par Gallimard dans la «Bibliothèque des Histoires ", qui non seulement avait alors un rôle de vigie, mais indiquait aussi les chemins à suivre et les perspectives nouvelles. Charles-Olivier Carbonell a également fait beaucoup pour l'historiographie, en s'inscrivant, bien avant les années I980, dans le sillage d’Henri-Irénée Marrou. Mais son travail très précis de dépouillement et de mesure de ce qui pouvait relever de l'historiographie, me semble-t-il, n'a pas joui d'une si large réception. Hormis, précisément, au début des années I980, lorsqu'il a fait par exemple paraître, en I98I, un “Que sais-je? » sur l'historiographie ${ }^{8}$. C'est également un signe aussi: si les Presses universitaires de France, qui avaient un rôle central, à cette époque, dans tout ce qui était le champ universitaire, consacre un “Que sais-je? » à l'historiographie, cela signifie qu'il y a un «marché ».

Ensuite, il y a eu cette autre interrogation sur l'histoire, via le travail de Michel de Certeau. Ce qui n'est pas la même chose. Dans un cas, l'historiographie, telle que la pratiquait Momigliano, c'est l'histoire de l'histoire, qui a une longue tradition derrière elle. Dans l'autre cas, l'historiographie, au sens proposé par Michel de Certeau, renvoie à la réflexion sur l'écriture de l'histoire. Ces deux conceptions peuvent trouver leur place dans un ensemble que l'on appelle «historiographie», mais le questionnement n'est pas le même. On peut pratiquer une interrogation sur l'écriture de l'histoire sans nécessairement s'intéresser véritablement à une histoire de l'histoire. La conjonction, au fond, des deux approches et peut-être les quiproquos produits par cette conjonction ont conduit à une certaine visibilité de l'historiographie entre les années ig8o à 2000. Cette visibilité s'est traduite, en matière institutionnelle, par l'introduction dans certains enseignements des universités d'unités de méthodologie et d'historiographie, qui témoigne d'une volonté de faire prendre conscience aux étudiants que l'histoire a une histoire et qu'elle n'est pas simplement un ensemble déjà constitué, qu'il faut apprendre de la manière la plus précise possible.

7 A. Momigliano, Problèmes d'historiographie ancienne et moderne, Paris, Gallimard, Ig83.

8 Ch.-O. Carbonell, L'historiographie, Paris, PuF, “Que sais-je? », I98I. 
Si j'élargis encore un peu la perspective: pour quelles raisons ces travaux ontils pu trouver place chez les éditeurs et dans les cursus universitaires au cours de ces années-là? Je pense qu'il faut rapporter ce phénomène au changement de conjoncture dont nous faisions l'expérience: un changement de notre rapport au temps. Au fond, l'historiographie participe de ce moment où, en différents lieux et dans différentes institutions, on s'interroge sur le lieu d'où l'on vient et sur ce qui fait que l'on en est là aujourd'hui. C'est le moment du grand développement des recherches généalogiques, porté par l'engouement du grand public et les transformations que connaissent alors les archives départementales pour répondre à cette demande nouvelle. C'est aussi le moment où un certain nombre d'entreprises se préoccupent tout d'un coup de leurs archives - dont elles se débarrassaient jusqu'alors allègrement -, en créant ou en développant leurs propres services d'archives. Et, plus largement encore, c'est le début des “années patrimoine », avec cette interrogation sur les signes qui nous permettent de nous repérer et de nous reconnaître, parce que ces signes et ces repères ont déjà largement disparu, ou sont en train de disparaître. Je pense qu'il faut inscrire le développement de l'historiographie dans cette conjoncture.

Aujourd'hui, j'ai le sentiment - mais sans disposer d'éléments précis à faire valoir - que l'intérêt pour l'historiographie est largement retombé. Je ne sais si c'est une expérience que vous partagez ici, à Toulouse. J'ai néanmoins le sentiment que l'historiographie - qui a participé d'un moment ou d'un mouvement réflexif dans les sciences sociales - est désormais connue et intégrée dans les démarches des différentes disciplines, mais n'est plus une approche ou une perspective qui traverse profondément la discipline historique.

Pascal Payen: Je voudrais poursuivre sur un autre point, qui s’inscrit sinon dans la longue durée, du moins dans le temps long, et qui concerne ce que j’appellerais les outils de l'historien. En histoire, on peut mesurer les progrès à la découverte de nouveaux dossiers documentaires, de nouvelles sources, c'est entendu; mais on peut aussi mesurer les progrès à la lente mise au point de nouveaux outils d'analyse, destinés à explorer le champ de l'enquête historique. En ce domaine, il me semble, vos travaux ont fait progresser la science historique dans deux registres, qui ont trouvé leur aboutissement dans deux livres à propos desquels je souhaiterais avoir votre avis. Ces deux livres, ce sont Régimes d'historicité ${ }^{9}$ et Anciens, modernes, sauvages ${ }^{10}$, avec cet outil d'analyse que vous avez promu: le couple Anciens et Modernes. Pourriez-vous reconstituer l'émergence, dans votre

9 F. Hartog, Régimes d'historicité: présentisme et expériences du temps, Paris, Éditions du Seuil, 2012 $2^{2}$ [2003].

10 F. Hartog, Anciens, Modernes, Sauvages, Paris, Galaade, 2005. 
réflexion, de ce premier couple et dire, peut-être, si vous pensez que l'usage qui en a été fait reste fidèle à ce que vous avez proposé?

François Hartog: En ce qui concerne les Anciens et les Modernes - qui sont également présents dans Partir pour la Grèce ${ }^{11}$-, j’ai expliqué comment ce couple venait prendre place dans une série de couples apparus antérieurement, sans pour autant modifier la façon dont ces couples antérieurs fonctionnaient. Ces couples antérieurs, ce sont “païens et chrétiens » et “Grecs et barbares ». Anciens, modernes, sauvages m'a amené à essayer de saisir la constitution du couple Anciens et Modernes, son efficace en termes intellectuels, cognitifs et politiques, dans la longue durée de l'histoire et de la culture européennes. Mais ce qui m’a le plus intéressé, ce sont les moments de crise et de mise en question du couple, et le moment de crise le plus patent - sachant que l'histoire même du couple est une histoire de confrontations et de querelles plus ou moins vives - est l'apparition d'un troisième terme, à savoir le sauvage. C'est la perturbation introduite par le sauvage, avec la découverte du Nouveau Monde, que j’ai essayé de pointer. L'introduction du sauvage déstabilise la relation entre l'Ancien et le Moderne, ou entre l'Ancien et le civilisé. Celui qui présente une épure de cette situation, c'est Montaigne, dans les Essais, avec son fameux chapitre sur les cannibales ${ }^{12}$. Les Anciens, dans ce texte, sont rapprochés des sauvages, et il y a, à terme, un effet d'ensauvagement des Anciens. Les sauvages, pour Montaigne, viennent miner la position du Moderne ou du civilisé, avec ce retournement dont Montaigne n'est pas l'inventeur mais dont il use: l'introduction du sauvage vient fortement questionner le civilisé, puisque des deux, finalement, le plus barbare ou le plus sauvage, c'est le civilisé.

J'ai poursuivi cette interrogation sur les remises en question du couple, avec cet autre moment qu'on a appelé la Querelle des Anciens et des Modernes c'est-à-dire avec Charles Perrault et son ouvrage ${ }^{13}$. Ce moment très important est celui de l'utilisation de l'instrument cognitif très puissant qu'est le parallèle. Le couple Anciens et Modernes invite au parallèle, qui est le grand instrument de ce modèle d'histoire, fort ancien, qu'est l'histoire «maîtresse de vie (historia magistra vitae $)^{14}$. Or, le livre de Charles Perrault, Le parallèle des Anciens et des Modernes, est instructif puisqu'il démontre, précisément, que le parallèle n'est plus tenable. Autrement dit, il n'y a plus de parallèle possible entre les Anciens et les Modernes : la conclusion, au contraire, est celle de la supériorité des Modernes

1 F. Hartog, Partir pour la Grèce, Paris, Flammarion, 2015.

12 Montaigne, “Des Cannibales », Essais, I, 3I.

13 Ch. Perrault, Le parallèle des Anciens et des Modernes, Paris, 1688.

14 Cicéron, De Oratore, II, 36. 
sur les Anciens. On passe de la supériorité des Anciens sur les Modernes à la supériorité des Modernes sur les Anciens, tout en utilisant toujours officiellement le parallèle, désormais renversé. À partir de ce moment-là, le rapport des Anciens et des Modernes se trouve profondément modifié. Si je poursuis encore d'un pas, le moment, dans l'histoire de la France, de remise en question ou de rejet du couple des Anciens et des Modernes, c'est la Révolution française, qui marque la fin de l'usage de ce couple pour réfléchir au régime politique. Il ne faut plus aller chercher du côté des républiques anciennes des modèles ou même des références pour penser le régime possible de la France, car cet usage-là a été condamné par les excès de la Révolution, autrement dit par la Terreur. C'est ce moment, à la suite de Thermidor, de la position développée par les libéraux et théorisée par Benjamin Constant au début du xix ${ }^{\mathrm{e}}$ siècle. Dans sa célèbre conférence de i8ı sur La liberté des Anciens comparée à celle des Modernes, où il met en forme ces deux libertés : celle des Anciens, qui était toute de participation, et celle des Modernes, qui est toute de jouissance privée. Les Jacobins ont confondu les deux libertés, en voulant reprendre la liberté des Anciens dans un monde qui n'était plus celui des Anciens. C'est là, justement, la prise en considération du facteur temps: les temps ont changé, et on ne peut plus opérer cette confusion, sinon à conduire à des catastrophes. Ce parcours-là est le fil conducteur de ce livre.

Corinne Bonnet: Je reviens un peu en arrière sur la notion de « régimes d'historicité », qui est une notion centrale dans votre œuvre et qui vise à cibler les procédés complexes à travers lesquels on exprime et on construit une expérience du temps : comment articule-t-on le passé, le présent et l'avenir et, en même temps, comment articule-t-on le temps et l'espace? Vous donnez l'exemple des Maoris, qui considèrent que le futur est déjà physiquement derrière eux - les Assyriens, d'ailleurs, avaient déjà exactement la même conception. Donc “partir pour la Grèce», c'est effectuer un voyage à la fois dans le temps et dans l'espace. Or, première surprise, quand on lit le livre: en fait, on part pour la Grèce et pour Rome, d'emblée. À la première, suggérez-vous, va la “gloire», à la seconde, la “grandeur ${ }^{15}$ », notamment parce que Rome a servi à la Grèce de relais, et même, pourrait-on dire, de caisse de résonance. La première question que je voudrais vous poser est la suivante: comment peut-on ressaisir ou appréhender cette « conjoncture intellectuelle» qui relie la Grèce à Rome? Vous avez dit tout à l'heure que ce qui est important, ce sont notamment les passages. La formule de Paul Veyne, qui parle d'un «Empire grécoromain ${ }^{16}$ », est-elle la meilleure ou la plus pertinente pour décrire la continuité ou les discontinuités, subtiles et problématiques, qui les lient? Quels sont, en même

15 F. Навто, Partir pour la Grèce, op. cit., p. 9.

16 P. Veyne, L'Empire gréco-romain, Paris, Éditions du Seuil, 2005. 
temps, les filtres qui opèrent dans ce passage, dans cette transmission de conjoncture entre Grèce et Rome?

François Hartog: Je dirai que c'est au fond la même démarche pour moi: “la Grèce et nous », “les Grecs et nous» ou «nous et les Grecs». Pour essayer de saisir comment s'est construit ce rapport, il apparaît très vite qu'il faut introduire, là aussi, un troisième terme, à savoir les Romains. Le rapport entre les Grecs et nous - quel que soit ce «nous» - passe par la médiation des Romains. Si on veut comprendre comment s'est constitué l'objet Grèce pour les Modernes, il faut repartir de ce moment où les Romains se trouvent face au problème des Grecs, pour répondre à la question de leur identité: “Qu’est-ce qu'être romain? ». Les Grecs leur proposaient un choix, difficile pour eux, qui était celui du couple Grecs et barbares : “Sommes-nous des Grecs ou des barbares? ». Évidemment, ils n'avaient aucune raison de répondre «nous sommes des barbares », et répondre “nous sommes des Grecs» a tenté un certain nombre de Romains, pendant longtemps. Et les Romains ont produit, grâce à des intellectuels grecs aussi, toutes sortes de réflexions généalogiques, parce que les Anciens procédaient de cette manière. Une érudition gigantesque, d'une inventivité considérable, s'est développée autour de ces questions généalogiques. On pouvait trouver là une manière de rattacher les Romains aux Grecs. Mais ce n'est finalement pas la solution qui l'a emporté et, chez les Romains, ce qui est devenu la croyance commune, même si elle est produite dans un cercle intellectuel, c'est: “nous sommes des Troyens». Par conséquent, on échappe à l'alternative entre Grecs et barbares. «Nous sommes des Troyens et nous sommes en train de prendre notre revanche sur ces Grecs qui ont vaincu les Troyens ", puisqu'il y a eu - on ne la trouve pas, cependant, chez Homère - cette assignation des Achéens aux Grecs et des Troyens aux barbares. Ainsi, Hérodote raconte comment Xerxès alla en pèlerinage à Troie, pour revendiquer cet héritage ${ }^{17}$. Du point de vue cognitif, ce passage nécessaire par les Romains me semble la bonne voie pour suivre la construction de l'objet que nous, Modernes, avons appelé « Grèce classique».

Corinne Bonnet: Votre dernier livre se présente par son titre, mais également par son illustration - ce temple qui est une peinture de Lichtenstein du temple d'Apollon à Corinthe -, comme une invitation au voyage à travers ce qui semble un signe ou un label: la Grèce est forcément un temple. Cela m'invite à vous interroger sur ce qui n'est pas seulement «partir», avec cette charge d'attente, de rêve, d'illusion, mais aussi “arriver en Grèce», avec ce qu'une telle issue peut impliquer de désillusion, voire de confrontation avec une autre Grèce, la Grèce des

17 Hérodote, Histoires, VII, 42-43. 
Modernes. Au moment de l'arrivée en Grèce, on passe en quelque sorte du mythe à la réalité. Sur ce plan, je voudrais invoquer deux témoignages intéressants, que je vais mettre en confrontation. Ernest Renan part en i865 pour la Grèce, arrive à Athènes et décrit ses impressions dans sa fameuse Prière sur l’Acropole:

Ce fut à Athènes, en I865, que j'éprouvai pour la première fois un vif sentiment de retour en arrière [Renan part donc, mais revient en arrière dans le même temps], un effet comme celui d'une brise fraîche, pénétrante, venant de très loin. L’impression que me fit Athènes est de beaucoup la plus forte que j'aie jamais ressentie. Il y a un lieu où la perfection existe; il n'y en a pas deux: c'est celui-là [...]. Quand je vis l'Acropole, j'eus la révélation du divin, comme je l'avais eue la première fois que je sentis vivre l'Évangile, en apercevant la vallée du Jourdain des hauteurs de Casyoun. Le monde entier alors me parut barbare ${ }^{18}$.

Le second témoignage vient de Maurice Barrès, une toute autre personnalité, mais qui connaissait Renan bien sûr. Barrès visite la Grèce en Igoo et publie quelques années plus tard un Voyage de Sparte, où il choisit de mettre l'accent sur la cité laconienne, comme pour prendre une distance par rapport à Athènes et à l'étreinte mystique de Renan. Il écrit, en arrivant:

Ce petit rocher ruineux se rattache en nous à tant d'idées préalablement associées que ce seul mot des passagers: “Athènes! voici l'Acropole!» détermine dans ma conscience le même bruissement qu'un coup de vent dans les feuilles de la forêt. Mon jugement n'avait aucune part dans mon enthousiasme, car ce premier aspect d'Athènes, exactement, me déconcertait par son apparence de bibelot bizarre $[\ldots]^{19}$.

Deux façons, donc, d'arriver et de faire l'expérience de la Grèce. Quelle est, à cet égard, votre propre expérience de l'arrivée en Grèce? Êtes-vous du côté de l'“étreinte mystique» ou du “bibelot bizarre»? Par ailleurs, évoquer les conjonctures, c'est aussi évoquer la Grèce d'aujourd'hui. Comment, dès lors, intégrer dans nos démarches, dans nos savoirs, la Grèce d'aujourd'hui, Grèce touristique, Grèce de la crise, Grèce des réfugiés que l'on voit aujourd'hui quotidiennement à la télévision? En somme, comment interroger la Grèce antique au départ de la relation entre le passé et le présent, qui sans cesse se reconfigure?

François Hartog: Renan, pour dire un mot de lui, est ondoyant. Vous trouvez dans un texte une phrase qui dit ceci et vous en trouverez une autre ailleurs, ou un peu plus loin, qui dit tout autre chose. Par rapport à l'Acropole, à ce lieu du “miracle»-puisque c'est Renan qui parle du miracle d'Athènes, du miracle grec -, il y a un autre miracle, celui de Palestine. Cet autre miracle, lors du passage en

18 E. Renan, Souvenirs d'enfance et de jeunesse [I883], Paris, Gallimard, I983, p. 44-45.

19 M. Barrès, Le voyage de Sparte, Paris, Émile-Paul Éditeurs, Igo6, p. 43. 
Palestine au cours de la «mission de Phénicie», c’est l'invention du monothéisme par les Juifs. Il y a donc deux miracles. Ce qui, déjà, balance un peu les choses. Renan, par ailleurs, écrit toute sa Vie de Jésus sur le thème de l'inexistence du miracle. Dans la Prière sur l'Acropole, il joue beaucoup de l'opposition avec le barbare, puisque lui-même, venant des brumes du Nord - la Bretagne -, revendique une «barbarie» celtique. On a affaire à un jeu sur le champ sémantique du barbare, dans le texte de la prière. Et il s'achève, après la célébration du miracle divin qui le saisit alors qu'il se trouve sur l'Acropole, par le «linceul de pourpre où dorment les dieux morts ${ }^{20}$ ». C'est aussi une manière de dire que tout cela est fini. On ne peut donc pas simplement évoquer Renan et le miracle grec: c'est un miracle avec toutes sortes d'attendus.

Je ne l'ai pas fait intervenir dans mon livre, mais Ernest Renan est une figure très intéressante pour cette question du «partir pour la Grèce ». Lui, il est d'abord parti pour l'Orient: la Phénicie, avec en ligne de mire la Palestine, dont les paysages ont été pour lui comme un cinquième évangile. Il n'a eu la «révélation» d'Athènes, ce «lieu où la perfection existe», qu'ensuite, en I865, et, quand il écrira la “Prière sur l'Acropole », en ı876, il l'insérera au milieu de ses souvenirs bretons ! Vient ensuite le «bibelot bizarre d de Barrès. Dans son livre, qui a justement pour titre Le voyage de Sparte, il avoue son “désarroi » et sa “défiance » face à Athènes. Athéna n'est pas la raison universelle, mais une raison municipale. Alors que le “prodigieux haras » qu'a été Sparte, voulant produire une «humanité supérieure », l'enchante. À l'évidence, je ne suis pas du côté du miracle, moins encore de celui du haras! Pour moi, le départ vers ou le voyage effectif en Grèce est arrivé assez tard. Parce que nous avions décidé, avec quelques autres, que nous n'irions pas en Grèce aussi longtemps que les colonels seraient au pouvoir. Cela ne faisait certes pas grand-chose aux colonels que j'aille ou que je n'aille pas en Grèce, mais c'était une position de principe... Qui a eu pour effet direct qu'au moment où j'aurais pu aller en Grèce facilement, lorsque j'étais étudiant et qu'il était peu onéreux de s'y rendre, je ne l'ai pas fait. Mon rapport à la Grèce est donc d'abord passé par la médiation des livres et par des textes. Et on peut peut-être dire, au fond, que c'était déjà ce qui m’intéressait le plus. Je n'ai jamais eu la moindre envie d'aller à l'École française d'Athènes. J'aurais pu essayer et peut-être même m'aurait-on pris; mais, outre que je n'ai jamais eu d'attraits particuliers pour l'archéologie, mon intérêt pour la Grèce était plus d'ordre intellectuel que sensible. Des Grecs “bons à penser». Tout cela pour dire que mon rapport à la Grèce s'est construit sur cette distance. J'ai essayé, à partir de là, de faire de cette distance un ressort de mes interrogations sur l'objet Grèce. Par la suite, je suis allé effectivement en Grèce, j’y ai fait quelques voyages, peu nombreux cependant. Mais cela n’a jamais été un 
élément fort - même si me saisit toujours la beauté des îles vues de la mer - de mon rapport à la Grèce. Il ne s'est pas passé pour moi ce que raconte par exemple JeanPierre Vernant: lors de son premier voyage en Grèce, en ig3o - c'était au moment de la dictature de Metaxás, et il ne s'était pas posé la question de savoir s’il y allait ou n'y allait pas -, il a éprouvé un véritable émerveillement. Si vous avez eu l'occasion de lire l'Été grec de Lacarrière ${ }^{21}$, c'est de cette Grèce-là, au fond, que Vernant a fait l'expérience dans les années ig3o. J'aurais presque pu la connaître: dans les années I96o, la Grèce n'était pas encore l'usine à touristes qu'elle est devenue depuis, avec les immeubles de quinze étages qui vont d'escales en escales.

Adeline Grand-Clément: Nous avons parlé de la question du voyage, et c'est précisément l'un des points sur lesquels je souhaite revenir. Mes questions, comme les précédentes, toucheront à la fois au métier de l'historien et à votre itinéraire personnel, et permettront de comprendre comment ces deux dimensions peuvent se répondre dans votre cas. J'ai ainsi choisi de partir de deux livres, consacrés à deux figures qui ont peut-être été pour vous des compagnons de route, des compagnons de voyage dans votre itinéraire. Le premier de ces compagnons est Hérodote, que vous avez étudié notamment dans Le Miroir d'Hérodote. Essai sur la représentation de l'autre. La seconde figure, tout aussi emblématique, est celle d'Ulysse, évoquée dans un autre livre, Mémoire d'Ulysse. Récits sur la frontière en Grèce ancienne ${ }^{22}$. On remarquera, dans les deux cas, l'importance du sous-titre, qui complète et explicite les thèmes abordés dans ces essais. Parmi ces thèmes, qui informent aussi une large partie de vos travaux, on retrouve, tout d'abord, la question de l'identité et de l'altérité - et vous montrez bien, dans le premier ouvrage, comment la confrontation avec l'autre est un moyen de construire et d'affirmer une identité propre, à travers l'image du miroir et l'analyse des processus réflexifs. Le second thème concerne le voyage, la frontière, le déplacement, l'espace, qui occupent une place très importante. En vous écoutant, je me demandais si on ne pouvait pas définir l'historien comment un «arpenteur du temps », pour reprendre l'idée de déplacement.

Ce sont ces deux thèmes que je souhaiterais aborder avec vous. Je vous propose de partir, pour le premier thème, d'une citation de Paul Veyne à propos de l'histoire:

L'histoire peut être définie comme l'inventaire explicatif, non pas des hommes ni des sociétés, mais de ce qu'il y a de social en l'homme, ou plus précisément des différences que présente cet aspect social. Il suffit par exemple que la perception des couleurs soit

21 J. Lacarrière, L'Été grec: une Grèce quotidienne de 4000 ans, Paris, Plon, 1976.

22 F. Hartog, Mémoire d'Ulysse: récits sur la frontière en Grèce ancienne, Paris, Gallimard, I996. 
différente d'une société à l'autre (aux yeux des Grecs, la mer était violette); ipso facto les couleurs appartiendront à l'histoire autant qu'à la science ${ }^{23}$.

J'aime beaucoup cette citation, bien sûr parce qu'il est question de couleurs, mais aussi parce qu'il est question de la mer, cet espace qui permet la traversée et qui est également présent, en creux, dans nombre de vos études. Si l'on accepte la définition que propose Paul Veyne, le travail de l'historien se rapproche de celui de l'ethnologue ou de l'anthropologue. Vous avez parlé hier de Lévi-Strauss, qui compte parmi les figures qui vous ont influencé, accompagné, en évoquant notamment ce “regard éloigné » dans lequel vous vous reconnaissez, ce mouvement particulier de l'anthropologue qui reflète aussi, selon vous, le propre travail de l'historien. Si l'on peut admettre qu'il y a des analogies entre le travail de l'historien et celui de l'anthropologue de terrain, j'aimerais que l'on revienne aussi sur les différences qu'il peut y avoir entre ces deux approches. Qu'induit en particulier, dans le cas de l'helléniste, le fait que l'éloignement temporel se substitue à l'éloignement spatial? L'historien voyage certes lui aussi, mais dans le temps.

François Hartog: Les deux livres que vous évoquez ne cessent en effet de poser la question de l'espace et du temps. Je signale au passage que, très souvent, le livre Mémoire d'Ulysse est cité comme les mémoires d'Ulysse, comme si j'avais écrit les mémoires d'Ulysse, ce qui n'est évidemment pas mon propos... L'ouvrage, intitulé Mémoire d'Ulysse, au singulier, traite de la mémoire d'Ulysse dans la tradition, des usages, des reprises de la figure d'Ulysse, comme figure fondatrice, comme “homme-frontière ${ }^{24}$ ", c'est-à-dire comme celui qui, dans ses déplacements, dans ses errances, fait l'essai, à travers le récit homérique, de toutes les frontières qui définissent la condition humaine. Il fait l'essai des frontières avec le monde divin - la question de l'immortalité qui lui est proposée par Calypso; il fait l'essai de la frontière avec le monde des morts, dont il s'approche au plus près dans la Nekuia, au chant XI de l'Odyssée; il fait l'essai, enfin, de l'impossibilité de vivre dans une société autre que celle des «hommes mangeurs de pain », pour reprendre l'expression d'Homère. Voilà pourquoi Ulysse a une place centrale dans ce livre.

Mais il y a une autre raison, qui ne répond pas encore à votre question, mais que je peux évoquer. Cette figure réapparaît en effet dans mon livre Régimes d'historicité, où j'évoque cette scène, que j'appelle “primitive ${ }^{25}$ », de la rencontre avec les Phéaciens et des pleurs d'Ulysse. Scène fameuse ${ }^{26}$, au cours de laquelle Alcinoos demande à Ulysse ce qu'il souhaite que l'aède chante lors du banquet, comme on

23 P. Veyne, L'inventaire des différences, Paris, Éditions du Seuil, ı976, p.52-53.

24 F. Hartog, Mémoire d'Ulysse, op. cit., p. 12.

25 Ibid., p.79.

26 Homère, Odyssée, VIII, 492-498. 
le fait pour honorer un hôte. Ulysse répond qu'il souhaite écouter l'épisode du cheval de bois et de la prise de Troie, qui est son propre moment de gloire. Chez les Phéaciens, donc, Ulysse, qui n'a désormais plus de nom et n'est, à vrai dire, plus personne - songeons au fameux “Personne, voilà mon nom ${ }^{27}$ » -, demande ce chant, et Démodocos, aède qui remplit parfaitement son rôle, se met aussitôt à chanter cet épisode. Il le fait d'une manière aussi précise, aussi vivante, que s'il avait effectivement assisté à la scène ou entendu le récit d'un témoin direct, à tel point qu'Ulysse lui demande même s'il était présent à Troie. Or, Ulysse sait très bien que Démodocos n'était pas présent à Troie et qu'il est lui-même le seul témoin de la prise de la cité. On a ainsi, dans cette scène chez les Phéaciens, le moment presque fondateur de ce que sera la démarche de l'historien dans l'Antiquité, plus tard mise en forme par Hérodote: l'historien est celui qui ou bien voit de ses propres yeux - c'est l'«autopsie», au sens étymologique du terme -, ou bien entend, reçoit et recueille des récits, des logoi dits par d'autres, dont il essaie d'évaluer la vraisemblance. On a dans ces quelques vers de l'Odyssée un passage tout à fait étrange, qui laisse entrevoir ce qui devait devenir, plusieurs siècles plus tard, une définition du métier de l'historien. Ulysse sait très bien, comme tous les auditeurs présents, que Démodocos n'a pas assisté à la prise de Troie et que les véritables inspirateurs de son chant sont Apollon et les Muses, que l'aède, par définition, est celui qui possède la vision octroyée par les Muses. On se situe donc dans un tout autre registre que celui des yeux de chair, et la question d'Ulysse est d'autant plus invraisemblable que Démodocos est présenté comme étant aveugle. C'est l'excursus que je souhaitais faire sur ce qui allait devenir - ce qui aurait aussi très bien pu ne pas advenir -, la manière de faire de l'histoire, dès Hérodote.

Pour ce qui concerne la question de l'altérité, je dirais que mon livre, le Miroir d'Hérodote, s'inscrit, du point de vue de la conjoncture intellectuelle que nous avons évoquée, dans ce moment où cette question était très présente et où on raisonnait en termes d'altérité. Il s'agit donc, non d'un plaquage, je l'espère, mais d'une reprise de cette thématique, dans le but de lire Hérodote. Vous avez évoqué le nom de Lévi-Strauss. Hérodote, je l'ai souvent dit, et peut-être même écrit, est pour moi une sorte de “Lévi-Strauss de l'Antiquitée ${ }^{28}$ ). Je veux dire par là qu'il y a, chez Hérodote, un effort de mise en ordre, de mise en place du monde, des grands partages, de ce qui fait la proximité, la distance, l'altérité, ces degrés de proximité et d'éloignement, une carte culturelle du monde, de la même façon que Lévi-Strauss lui-même - songeons par exemple à l'analyse développée dans Tristes topiques (I955) ou dans le texte Race et culture (I97I), qui a eu un retentissement considérable - propose une représentation du monde et de la place des uns et des

27 Homère, Odyssée, IX, 366.

28 F. Навтоg, Mémoire d'Ulysse, op. cit., p.99. 
autres dans cet univers. C'est aussi la démarche d'Hérodote et ce à quoi aboutissent ses Histoires.

Adeline Grand-Clément: Quelles différences, précisément, peut-on observer entre le travail de l'anthropologue de terrain et celui de l'historien, en particulier du point de vue du rapport au temps et à l'espace?

François Hartog: Lévi-Strauss, en effet, a eu la possibilité de rendre visite aux Caduveo, aux Nambikwara, et d'élaborer, à partir de cette expérience, sa vision à la fois de ces populations et du monde européen, dans la distance avec ces sociétés menacées. L'historien est dans l'impossibilité de le faire. Le contemporanéiste qui fait de l'histoire orale peut certes le faire, mais quand il s'agit d'histoire ancienne, d'histoire médiévale ou d'histoire moderne, le problème se pose dans des termes différents. C'est là qu'intervient la réflexion historiographique, puisque l'historien doit lutter contre cette tendance qui consiste à considérer que, dès lors qu'il dispose d'un document, il détient une réponse à sa question, qu'il lui suffit de lire un document, de le transcrire et d'écrire l'histoire de tel ou tel objet. La réflexion historiographique permet précisément la prise de conscience que le temps est un facteur pertinent. Entre l'historien et le document interviennent toute une série de conjonctures, politiques, intellectuelles, culturelles, qui font que sa lecture ne peut pas ne pas être modifiée par le passage du temps et par les transformations qu'il apporte. Il y a donc une évaluation très fine à faire, qui permet de nuancer, de reformuler le sens que l'on avait cru pouvoir déceler, à première vue, dans le document. La première règle du travail de l'historien est précisément de se méfier du «à première vue».

Adeline Grand-Clément: Je vous propose d'aborder le second thème, celui du voyage. À la question “Comment devient-on historien? », on pourrait peut-être répondre: “En voyageant». Si l’on songe aux figures que vous avez étudiées, aux historiens ou aux intellectuels qui vous ont marqué, on a souvent affaire à des voyageurs: je pense à l'exil contraint d'Hérodote, qui quitte Halicarnasse pour arpenter une partie du Bassin méditerranéen et de l'Asie; à l'échec de Thucydide à Amphipolis et à la condamnation à l'exil qui s'ensuit; à l'exil de Momigliano, contraint de quitter l'Italie en Ig38; à Lévi-Strauss, écrivant pourtant au seuil de Tristes Tropiques: “Je hais les voyages et les explorateurs ${ }^{29}$. C Comme si le voyage était nécessaire, parce qu'il permet d'accéder, pour reprendre votre analyse, à cet “espace d'entre-deux ${ }^{30}$ », parce qu'il permet, pour le dire avec Vernant, une

29 C. Lévi-Strauss, Tristes Tropiques, Paris, Plon, I955, p. I3.

30 F. Hartog, Mémoire d'Ulysse, op. cit., p.2I. 
“traversée des frontières ${ }^{31}$ ». Estimez-vous qu'il y a effectivement, au départ du projet de l'historien, un voyage? Quelles formes a prises le voyage pour vous? J'ai le sentiment que, dans votre cas, c'est peut-être d'abord par les textes que passe le voyage. Sous toutes ses formes, le déplacement est-il inhérent, selon vous, au travail de l'historien?

François Hartog : Je répondrai : oui, il faut voyager, de toutes les façons possibles, et surtout ne pas rester sur place. En ce qui me concerne, vous avez évoqué les voyages dans les textes, et j’en ai, en effet, moi-même parlé. Mais si je peux évoquer un détail biographique, j'ai commencé par faire un voyage effectif, pendant un an, autour du monde. J'ai eu la chance de bénéficier d'une bourse, et j'ai notamment voyagé en bateau; j’ai passé près de six mois dans l'archipel indonésien, où l'on naviguait encore beaucoup à la voile. Pour moi qui ne parlais pas les différentes langues de l'Indonésie, la navigation, le contact avec les marins, les pêcheurs, les chantiers navals, étaient aussi un moyen de pouvoir rencontrer des gens. Du point de vue de mon travail - je venais juste de déposer, avant de partir pour un an, un sujet de thèse -, j'ai eu sur place la tentation de l'ethnologie. Pourquoi, me suis-je dit, plutôt que de travailler sur les Grecs, ne pas me lancer dans l'ethnologie de l'Indonésie? J'ai même eu l'occasion, sur place, d'échanger avec un ethnologue sur ce sujet, mais je ne l'ai finalement pas fait. Peut-être par manque d'audace, puisque cela impliquait, malgré tout, d'apprendre beaucoup de choses nouvelles; peut-être par un insuffisant désir de voyage. Ce qui est sûr, en revanche, c'est que mon approche de ce qu'était initialement mon sujet de thèse s'est trouvée complètement modifiée. Hérodote faisait certes partie de mon travail au départ, mais celui-ci ne lui était pas entièrement consacré; dans sa première formulation, mon sujet de thèse, qui m’avait été suggéré par Moses Finley, devait me permettre de reprendre la question de la «nation " grecque et des multiples appartenances du citoyen grec. Il s'agissait donc d'un sujet “gréco-grec», si je puis dire, davantage qu'un sujet portant sur les rapports entre Grecs et barbares. Ce n'est qu'après ce voyage que mon travail est devenu Le Miroir d'Hérodote. Le voyage n'a donc pas seulement été un voyage dans Hérodote. Je pense que cette approche, pour moi, est demeurée constante. Elle a pris des formes différentes, mais je crois fortement aux vertus de l'écart, du décentrement, du départ, qui permettent de regarder autrement ce qui, étant trop familier, ne se voit pas.

Manon Champier: Mes questions porteront sur la place de l'historien aujourd'hui. C'est un aspect que vous avez notamment abordé dans Régimes d'historicité, en confrontant les pratiques actuelles de l'histoire avec le «présentisme»

31 J.-P. Vernant, La Traversée des frontières, Paris, Éditions du Seuil, 2004. 
de nos sociétés. En évoquant la notion de présentisme, vous avez souligné le rôle des médias dans l'omniprésence de ce que vous avez également appelé le «courttermisme». En 200I, vous avez codirigé, avec Jacques Revel, un ouvrage sur Les usages politiques du passé, dans lequel vous soulignez le fait que «le débat historique et, plus largement, les différentes formes de modification du passé ne sont plus confinés dans un cercle de spécialistes ${ }^{32}$ ». En 20I2, dans la préface de la réédition de Régimes d'historicité, vous avez également souligné la capacité de l'historien à effectuer une “prise de recul », une « déprise du présent, grâce à la pratique du regard éloigné », “à créer de la distance pour, au terme de l’opération, mieux voir le proche ${ }^{33}$ ». Comment cette attitude pourrait-elle se traduire, si l'on songe par exemple au rôle qu'on attribue parfois à l'histoire, aujourd'hui, dans l'analyse d'un phénomène d'actualité comme celui des migrants? Quelle peut être la place de l'histoire et de l'historien dans les débats du temps présent?

François Hartog: Vous avez évoqué les Régimes d'historicité et la question de savoir ce que devient, dans un moment où la catégorie du présent est la catégorie dominante, prépondérante, le rôle de l'historien, lui qui s'est défini au xix ${ }^{\mathrm{e}}$ siècle comme un pontife, capable, selon le mot de Gabriel Monod ${ }^{34}$, d'établir des liens entre le passé et le présent, entre la «vieille France» et la «France moderne». Dès lors qu'il n'y a plus de passé, pour schématiser à l'extrême, que peut raconter l'historien? C'est en effet une question importante. Je dirais, en revenant quelque peu en arrière, que l'histoire moderne, le concept moderne d'histoire, tel qu'il s'est cristallisé au cours du Xix ${ }^{e}$ siècle, affirme que l'histoire est en réalité "futuriste», qu'elle est tournée vers le futur; qu'elle est là pour comprendre ce qui se passe dans le présent et du même coup rendre perceptible le chemin qu'il convient de suivre pour aller vers le futur. L'histoire du xix ${ }^{\mathrm{e}}$ siècle, sous la forme des histoires nationales, est une histoire téléologique, écrite en direction du futur. On évalue alors l'état de la nation, par exemple, à la lumière de l'accomplissement que l'on voit au terme du processus. On a là affaire à une histoire-processus, dont le temps est un facteur important. À partir du moment où ce paradigme-là n'est plus opératoire, à partir du moment où le futur s'est fermé, ou en tout cas n'est plus porteur, quelle histoire peut-on écrire? La réponse à cette question a été, dans la conjoncture qui se manifeste à partir des années I980, la montée de la mémoire, qui prend de plus en plus d'importance et de place, dans l'espace public mais aussi dans les

32 F. Hartog, J. Revel, “Avant-propos », in Les usages politiques du passé, Paris, ehess, 200I, p.7.

33 F. Hartog, Régimes d'historicité, op. cit., p. I3.

34 G. Monod, “Introduction. Du progrès des études historiques en France depuis le Xvi ${ }^{\mathrm{e}}$ siècle », Revue historique I (I876), p.5-38, p. 38. 
travaux des historiens. Cette montée de la mémoire a conduit à ce qu'on a appelé une histoire de la mémoire. Jusqu'alors, les historiens récusaient la mémoire comme n'étant pas l'objet de leur travail; l'histoire, jugeait-on, se fait avec des documents, et commence là où la mémoire s'arrête. Avec la montée de la mémoire, qui vient contester l'histoire et lui reproche de ne pas avoir pris en compte tel ou tel événement, telle ou telle minorité, l’historien a été obligé de lui faire une place. Il a cependant essayé de le faire en montrant qu'on pouvait faire de l'histoire de la mémoire. C'était une manière de reprendre la main sur cette conjoncture; il s'agissait d'une réponse très pragmatique, très disciplinaire, d'une réponse de défense. Mais cela n'a pas suffi pour répondre à cette conjoncture marquée par le «présentisme », cette modalité de rapport au temps qui est celle de notre contemporain.

Cela nous conduit à la question des régimes d'historicité, en d'autres termes, des façons dont s'articulent les catégories du passé, du présent et du futur. Cette notion ne renvoie pas à un objet naturel, mais constitue plutôt un instrument heuristique, un artefact, relevant de l'idéal-type weberien, qui permet de saisir les moments où les choses changent, basculent, où les écarts se creusent et où les renversements s'opèrent, les moments de “crise» du temps, de crise dans le rapport au temps et dans l'expérience du temps. Nos sociétés ont vécu, entre I789 et 1989 , pour retenir deux dates symboliques, dans ce que j’ai appelé le «régime moderne d'historicité », c'est-à-dire dans ce régime où le futur devient la catégorie rectrice, motrice. Antérieurement régnait ce que j'ai appelé, sans originalité particulière, l'“ ancien régime d'historicité », dans lequel le passé était la catégorie dominante. C'est le modèle cicéronien de l'historia magistra vitae: pour comprendre le présent et agir sur lui, on commence par regarder du côté du passé ; on importe, du passé dans le présent, des exemples et des leçons. La grande machinerie de l'historia magistra vitae se met en place dans l'Antiquité, peut-être dès le rv siècle av. J.-C. - on pourrait affirmer que l'œuvre de Thucydide dans sa volonté de se présenter comme un «acquis pour toujours » participe de cet ordre-là -, et devait durer en Europe, non sans remises en question et transformations, jusqu'au xvıII ${ }^{\mathrm{e}}$ siècle. On passe donc, au cours du XvıII ${ }^{\mathrm{e}}$ siècle, de la prépondérance de la catégorie du passé à celle de la catégorie du futur, d'un futur certes non-advenu, mais qui éclaire désormais le passé et le présent et à partir duquel on retrace notamment l'histoire de la nation depuis ses origines. Par conséquent, dans ce rapport au futur, on voit clair dans le passé; le futur détermine ce qu'on peut conserver ou oublier du passé pour écrire l'histoire. À partir du moment où la clarté du futur s'estompe, il ne reste plus que le “présentisme», c'est-à-dire un présent qui se réduit au regard qu’il porte sur lui-même, qui n'a plus les points de vue extérieurs que sont le passé et le futur, mais qui fabrique lui-même, à chaque seconde, le passé et le futur dont il se sert.

Que deviennent la place et le rôle de l'historien dans cette situation? Tout d'abord, je n'ai bien sûr aucune légitimité pour me prononcer au nom de l'histoire, et les historiens font ce qu'ils veulent. La première chose à faire cependant, 
me semble-t-il, est de prendre conscience de cette situation nouvelle, particulièrement en Europe. Il s'agit ensuite de se demander, à partir de ce diagnostic, ce que peut signifier écrire l'histoire dans cette conjoncture. Je vais publier dans un prochain numéro du Débat un article intitulé “Vers une nouvelle condition historique ${ }^{35}$ », dans lequel je pose précisément la question de savoir si nous ne sommes pas entrés dans une nouvelle condition historique. Faut-il renoncer à l'histoire et admettre que la mémoire a réponse à tout? Faut-il essayer de réfléchir à un nouveau concept d'histoire? À la première question, je répondrai par la négative: la mémoire peut faire et fait beaucoup de choses; elle est une façon de convoquer du passé - souvent un épisode désastreux, criminel ou traumatique - dans le présent, mais elle n'ouvre pas sur le futur (sauf sur le mode du devoir de mémoire). Du même coup, la mémoire peut être très active, être à l'origine de commémorations, de politiques «mémorielles», mais elle permet surtout de dire “jamais plus» et de favoriser la reconnaissance des victimes et de leurs droits dans l'espace public. Et, aujourd'hui, par un glissement qui s'est opéré, d'héroïser les victimes. Or les victimes, quand elles en ont réchappé, sont des personnes pour lesquelles le temps s'est arrêté, au moment du traumatisme. Pour ces personnes on le voit bien, par exemple, dans le phénomène de la “justice transitionnelle» et des “ commissions de vérité et de réconciliation », dont celle d'Afrique du Sud a été le prototype et la référence -, il n'y a au fond que du présent, un temps suspendu qui n'est que présent. Tout l'enjeu, pour ces commissions, est de remettre le temps «en marche».

Que peut faire l'histoire? Elle peut feindre d'ignorer ce phénomène et continuer, forte de son organisation et de son fonctionnement comme discipline; ce n'est peut-être pas, toutefois, la meilleure façon de procéder. Autre réponse, proposée aujourd'hui: la réponse de l'histoire “globale». On a vu se développer, depuis une quinzaine d'années et après un temps d'hésitation sur la terminologie - histoire «mondiale», histoire “ universelle» -, une histoire globale, qui l'a finalement emporté. Certains ont pu regretter, dans cette perspective, le retard de la France dans ce domaine, son “provincialisme». Néanmoins, l'histoire globale dispose désormais d'une chaire au Collège de France; il y a aujourd'hui un certain nombre d'historiens qui se définissent comme des historiens «globaux» et sont vus comme des historiens de référence dans la profession. Reste cependant une question: l'histoire globale n'est-elle, au fond, qu'une nouvelle spécialité, une nouvelle pièce dans la «maison» Histoire, ou bien une reformulation, une refondation de cette même maison? Il me semble que les choses ne sont pas claires sur ce point, ou pas encore clarifiées. Quand on lit la leçon inaugurale de Sanjay

35 F. НавтоG, “Vers une nouvelle condition historique », Le Débat, à paraître. 
Subrahmanyam $^{36}$, élu en 2013 professeur d'histoire globale au Collège de France, il est clair que, pour lui, l'histoire globale est une spécialité nouvelle, mais qui n'a pas vocation à phagocyter les spécialités déjà établies. On a parfois l’impression que d'autres, au contraire, considèrent que la seule manière de faire de l'histoire est désormais l'histoire globale, qui deviendrait le concept même d'histoire pour le $\mathrm{xxI}^{\mathrm{e}}$ siècle. Mais, abstraction faite des réussites effectives de l'histoire globale, voiton émerger un nouveau concept d'histoire qui puisse répondre à la question du rapport au temps? Car si le concept d'histoire ne prend pas en charge l'analyse des manières d'articuler passé, présent et futur, il reste au fond un concept vide. Sur ce point, je ne lis pas tout ce qui paraît dans le domaine de l'histoire globale, devenu une véritable industrie, dont la langue de production est d'abord l'anglais, mais je n'ai pas perçu de formulation qui réponde à cette exigence: comment écrire l'histoire dans un monde devenu avant tout présentiste? La seule proposition que je puisse faire, compte tenu de ce que je sais faire, est la suivante: essayons de saisir les différentes temporalités à l'œuvre dans ce présent présentiste, en mobilisant la notion de «simultané du non-simultané ${ }^{37}$ », ou de “contemporain du non-contemporain »: le présent présentiste, que l'on voit généralement comme un bloc, est en réalité traversé par une pluralité de temporalités. Je ne prendrai qu'un exemple: le présentisme n'est pas uniforme, comme je l'ai suggéré dans la préface de la réédition des Régimes d'historicité ; il y a d'un côté un présentisme choisi, et généralement valorisé, celui de l'accélération, des flux, de la mobilité toujours plus grande, de l'instantanéité; mais il y a aussi un présentisme contraint, celui que subissent tous ceux qui, pour des raisons diverses, se trouvent dans l'incapacité de faire un projet: les chômeurs, les jeunes sans emploi ou avec des emplois précaires, les victimes de ce que Robert Castel a appelé le “précariat », de la précarité instituée les postdocs, par exemple, dans le domaine académique -, mais aussi les réfugiés, les émigrés, les déplacés, toutes ces personnes qui se comptent par millions. Ces catégories de populations n'ont que le temps présent et ne peuvent vivre qu'au jour le jour, dans le présentisme le plus extrême. Le «simultané du non-simultané » décrit bien la confrontation de ces deux présentismes : tous se trouvent dans un même présent, mais cette simultanéité ne signifie pas qu'il y ait simultanéité et concordance des temporalités dans lesquels ils vivent ou survivent.

Jean-Claude Carrière: Ma question se rapporte à l'une des conjonctures que nous avons évoquées et au développement du structuralisme. On a l'impression que le structuralisme s'est accompagné du développement d'une « non-historicité ».

36 S. Subrahmanyam, Aux origines de l'histoire globale, Paris, Fayard, 2014.

37 Voir «Le simultané du non-simultané. Entretien avec François Hartog », Europe 9I-20o9 (2013), p. 16-24. 
Jean-Pierre Vernant, par exemple, dans son fameux article sur le mythe des races d'Hésiode ${ }^{38}$, sorte de manifeste parfois critiqué au moment de sa publication, notamment par les spécialistes de la Sorbonne, ne consacre qu'une seule page à la signification sociale de son objet - de manière assez étonnante, du reste, pour un historien qui avait eu une expérience marxiste. Il ne s'interroge pas, par exemple, sur l'émergence de la notion occidentale d'État, sur l'idée d'une justice identique pour toutes les couches de la société. Après le structuralisme, marqué par ce régime de non-historicité, on en est venu à une réflexion sur l'histoire, centrée sur la question de savoir comment on écrit l'histoire. Passé ce deuxième moment, qui a duré un certain temps, on se demande aujourd'hui où nous en sommes. Vous venez de parler de l'histoire globale, mais quelle est, dans la conception actuelle de l'histoire, la part de l'anthropologie, de l'ethnologie? S'agit-il de faire de l'histoire des identités culturelles, dans leur étrangeté ou leur métissage? Quelle place reste-t-il aux problèmes traditionnels de l'histoire économique et sociale, issues en partie de la tradition marxiste? Font-ils toujours partie des préoccupations des historiens? Qu'est-ce, en définitive, que l'histoire globale? Quels outils anthropologiques ou ethnologiques, quels documents utilise-t-elle?

François Hartog: Un mot, tout d'abord, sur le structuralisme. Il y a eu, notamment de la part des marxistes, une lecture du structuralisme comme abandon de l'histoire. Si on lit Lévi-Strauss, cependant, qui a été en son temps accusé de tourner le dos à l'histoire, il ne l'a en fait jamais abandonnée. On a donc affaire à une déformation du structuralisme, même s'il y a eu en effet, parmi ceux qui ont pu s'en réclamer, une volonté de se débarrasser de l'histoire.

En ce qui concerne l'histoire globale, je ne suis pas un spécialiste, mais il y a un point qu'on peut souligner: nous sommes dans un moment de valorisation du travail d'archives, avec parfois l'idée, précisément, que tout se trouve dans les archives. L'histoire globale, elle, s'est largement fondée sur ce que ses praticiens ont appelé une histoire “connectée», considérée comme une manière de sortir d'une histoire “européo-centrée». L'histoire connectée s'inscrit dans le vaste mouvement de l'histoire postcoloniale, qui vise à rétablir de la symétrie dans l'histoire. L'histoire globale est au fond une généralisation de cette manière de faire de l'histoire. Romain Bertrand, un des grands spécialistes français, a écrit un livre sur l'Histoire à parts égales ${ }^{39}$ - en l'occurrence sur la rencontre, ou plutôt la

38 J.-P. Vernant, “Le mythe hésiodique des races. Essai d'analyse structurale» [I96o], in Mythe et Pensée chez les Grecs. Études de psychologie historique, Paris, La Découverte, I996 [1965], p. I9-47.

39 R. Bertrand, L'histoire à parts égales. Récits d'une rencontre Orient-Occident (xVI-XVII siècle), Paris, Éditions du Seuil, 201 . 
non-rencontre, entre les Hollandais et les royaumes javanais. À partir d'un travail d'archives, incluant les archives javanaises, il montre qu'on peut faire une histoire à parts égales, et que la grande affaire de l'arrivée des Hollandais n'était en fait qu'à peine mentionnée dans les archives locales. Dans ce cas, le souci de rétablir de la symétrie fait percevoir la dissymétrie. Il me semble toutefois que l'idée de symétrie est en définitive au fondement même du travail de l'historien. Songeons à la

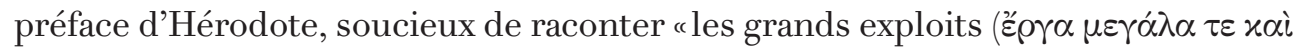
$\theta \omega \mu \alpha \sigma \tau \alpha$ ) accomplis tant par les Grecs, que par les barbares ${ }^{40}$ ». On peut discuter du traitement que propose Hérodote, mais là, déjà, l'histoire - et c'est ce en quoi elle se distingue de la mémoire - est décrite comme cette pratique fondée sur l'exigence, intellectuelle et éthique, de prendre en compte les deux côtés.

40 Hérodote, Histoires, I, I. 